\title{
Arthroscopic Resection of Humeral Enchondroma
}

\author{
Pedro Ferreira Pereira José Fernandes António Sousa \\ Manuel Gutierres \\ Serviço de Ortopedia e Traumatologia, Centro Hospitalar e Universitário de São João, Porto, \\ Portugal
}

\author{
Keywords \\ Enchondroma · Bone tumor · Shoulder arthroscopy · Curettage
}

\begin{abstract}
Enchondromas are common benign bone tumors. They are often found incidentally and usually do not require any treatment other than clinical surveillance. Signs of lesion progression or nontolerable pain are criteria for surgical resection. We present a case of a 44-year-old woman with long-lasting shoulder pain, diagnosed with enchondroma. Imaging studies showed an enchondroma near the great tuberosity. Shoulder arthroscopy made it possible to diagnose and treat an SLAP lesion as well as to resect by curettage and shaving through the subacromial lateral portal. One year after surgery, the patient had significant pain relief and improved shoulder function. Shoulder arthroscopy seems to be a safe and suitable technique for resection of small-sized humeral enchondromas, with the advantage of allowing to treat other possible concomitant shoulder pathologies.
\end{abstract}

\section{Introduction}

Enchondromas are benign, cartilaginous tumors, which represent about $3 \%$ of bone tumors and $13 \%$ of benign bone tumors [1]. They consist of masses of hyaline cartilage, usually found in the metaphyseal region of tubular bones in the hand, although they are also frequently found in the humerus or femur [2].

Enchondromas are commonly found incidentally on radiographs or other kind of imaging. They may present in any time of life, but are mainly found between the second and fourth

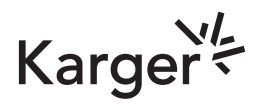


decades of life, and are often asymptomatic [1]. However, some cases may present with unspecific pain or some kind of swelling [3].

In asymptomatic and nonprogressive lesions, clinical and radiological follow-up seems to be the best attitude [4]. However, when signs of aggressiveness are present, some authors suggest surgical treatment [5]. The gold standard surgical treatment for enchondromas is intralesional curettage and filling the defect with bone graft or bone substitutes [6]. We present a clinical case of proximal humerus enchondroma resection through shoulder arthroscopy.

\section{Case Report}

A 44-year-old woman was referred to our hospital due to long-lasting right shoulder pain. Pain was unspecific, and the patient did not associate the pain with any particular activity. There was no history of previous shoulder trauma. In examination, her active shoulder motion was $120^{\circ}$ in anterior flexion, $90^{\circ}$ in abduction, $60^{\circ}$ in external rotation, and at the T8 in internal rotation. Radiograph study and MRI revealed a well-circumscribed lobulated, chondroid lesion at the proximal humerus, near the great tuberosity, without cortex invasion or any other sign of malignancy, compatible with enchondroma (Fig. 1). The patient was kept in
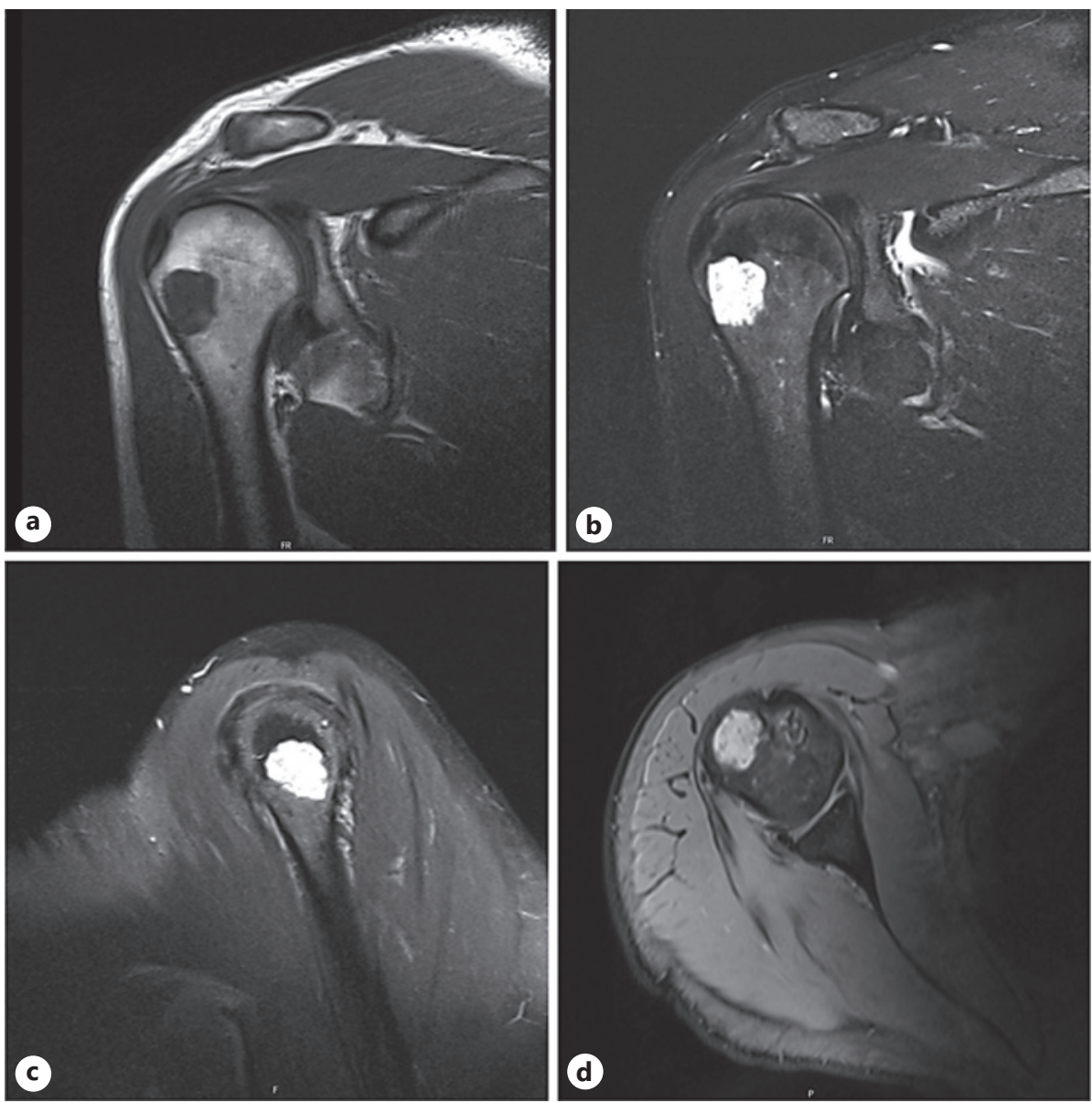

Fig. 1. MRI. a Low-intensity signal in T1-weighed sequences. b-d Coronal, sagittal, and axial cuts with highsignal intensity lesion on T2-weighted. 
Case Reports in Orthopedic Research
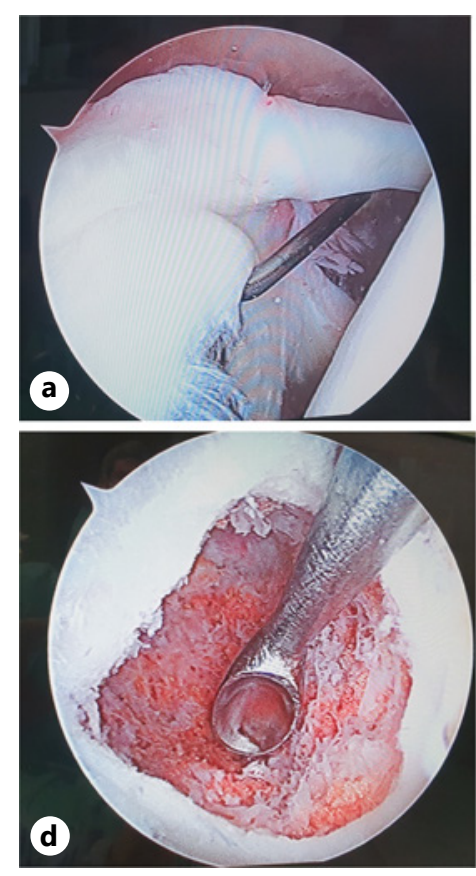

Fig. 2. a SLAP lesion. b, c Enchondroma in the humeral head and localizing using fluoroscopic guidance. d, e Intralesional resection using shaving and curettage. $\mathbf{f}$ Closure of portals.

Fig. 3. Radiograph 6 months after surgery showing no signs of recurrence.

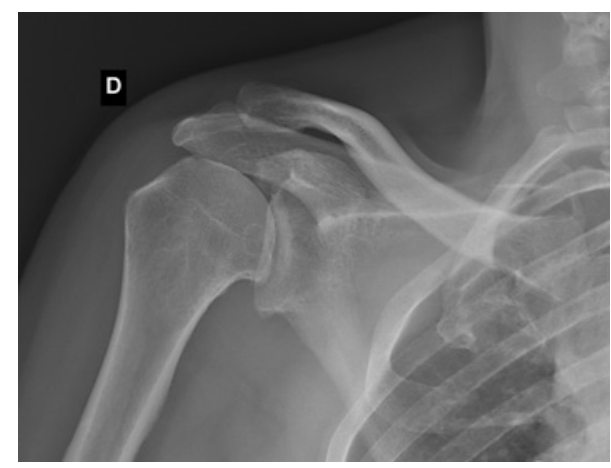

vigilance and treated with analgesic medications. Two additional MRIs were performed in 18 months that showed no sign of lesion progression or malignancy.

However, the patient maintained shoulder pain. Shoulder arthroscopy was performed for surgical treatment of enchondroma. Standard posterior and anterior portals were used. During inspection, a Snyder's type 2 SLAP lesion was found, and biceps tenotomy was performed. Then, using fluoroscopic assistance, the enchondroma was localized. Two additional lateral subacromial portals were used to reach the lesion which was resected with shaving and curettage. As it was not a big bone defect, only local bone graft was used, harvested from the humeral medullary cavity, through the lateral portal, with the curette (Fig. 2).

The postoperative period was uneventful. The patient was immobilized with a shoulder orthosis for 2 weeks. Histological analysis revealed mature hyaline cartilage with a lobular pattern, small and round chondrocytes, and no atypia, which confirmed the enchondroma.

Six months after surgery, the patient had significant pain relief and normal shoulder function. She improved mainly in shoulder anterior flexion and has no limitation at her job. Radiograph shows adequate bone healing after curettage and no signs of recurrence (Fig. 3). 


\section{Case Reports in Orthopedic Research}

\section{Discussion}

Enchondromas are common benign tumors. Classically, its most common location are the tubular bones of the hand. However, with more widespread use of MRI, it has been shown to be more frequent in the humerus and femur than previously reported. This can be explained because enchondromas are more easily seen on radiographs of smaller bones such as in the hand than on radiographs of the shoulder, hip, or knee [2]. A study with 477 shoulder MRIs found that the prevalence of incidental enchondroma was $2.1 \%$, well above what was previously reported [7].

Distinction between enchondroma and atypical cartilage tumors (ACT) is challenging and sometimes not possible. Clinically, both tumors are usually asymptomatic, although it has been reported that pain is more commonly found in ACT $[8,9]$. Previous reports pointed that radiological findings such as poorly defined margins, lesions with $5 \mathrm{~cm}$ or more, endosteal scalloping, and cortical thickening are in favor of ACT $[10,11]$. However, other authors stated that is often not possible to distinguish enchondroma from ACT based on radiological images [12]. Histologically, high cellularity, marked nuclear pleomorphism, and irregular distribution of cells are the features that point to the diagnosis of ACT [13]. There is no consensus regarding the treatment of enchondroma neither ACT. The majority of enchondromas does not need any treatment other than clinical and imaging surveillance [4]. However, surgical excision is indicated in cases of clinical or radiological aggressiveness such as endosteal scalloping or soft tissue extension, lesion growth or tumors bigger than $6 \mathrm{~cm}$, and untreatable pain $[3,5]$. Although extralesional resection has the highest oncological safety, intralesional resection with curettage to treat either enchondromas or ACTs in long bones has good oncological safety and excellent functional outcomes $[3,14]$. The cavity may be filled with autologous bone graft, which is biologically superior, or with bone cement that has the possible advantage to reduce recurrence rates due to heat destruction of the remaining tumor cells, which may be beneficial for large and radiologically aggressive enchondromas [5, 15].

To our knowledge, this is the first report of a humeral enchondroma resected by shoulder arthroscopy. Arthroscopic resection of enchondromas has been described in other anatomical locations such as the proximal femur or talus, with good results and adequate tumor resection $[16,17]$. Also, few cases are reported of resection of shoulder benign tumors through arthroscopy such as solitary bone cyst with osteoid osteoma $[18,19]$. In our case, shoulder arthroscopy allowed us to simultaneously diagnose and treat an SLAP lesion and perform an intralesional resection of the enchondroma. In fact, we believe that the main cause of symptoms of this patient was the SLAP lesion, since enchondromas of the proximal humerus are usually asymptomatic, and the pain is often attributable to another shoulder pathology [20]. We decided to do a biceps tenotomy instead of a tenodesis since our patient is a middle-aged woman, overweight, and not very demanding in her physical activities. Enchondroma was easily identified with fluoroscopic assist, and arthroscopy enabled an excellent visualization of the lesion as well as its resection by shaving and curettage. We used the bone of the medullary canal as graft to fill the defect, avoiding the morbidity of harvesting bone graft from another donor site. As in this case the bone defect after resection was small and in the metaphyseal area, without cortical thinning and only a minor cortical window was needed for curettage, humeral biomechanical stability was not compromised, and no osteosynthesis was needed.

Shoulder arthroscopy can be especially useful in painful shoulders with enchondromas. It is a minimal invasive surgery, with very few complications and less painful postoperative period, with which we can address other causes of shoulder pain and simultaneously resect the humeral enchondroma in case it is needed. We believe that as long as the lesion is small enough to not need osteosynthesis or a large bone grafting, it can be resected by arthroscopic intralesional shaving and curettage by experienced shoulder surgeons.

\section{Karger'}




\section{Conclusion}

At a time when we are seeing a strong evolution of minimally invasive surgery, shoulder arthroscopy seems to be a safe and suitable technique for resection of small-sized humeral enchondromas.

\section{Statement of Ethics}

The study is exempt from ethics committee approval, since it is a case report, and the patient has given her written informed consent for publishing data and images.

\section{Conflict of Interest Statement}

The authors have no conflicts of interest to declare.

\section{Funding Sources}

This research did not receive any specific grant from funding agencies in the public, commercial, or not-for-profit sectors.

\section{Author Contributions}

Pedro Ferreira Pereira, José Fernandes, and Manuel Gutierres participated in the treatment and follow-up of the patient as well as in the writing of the work. António Sousa reviewed and corrected the manuscript.

\section{Data Availability Statement}

All data generated or analyzed during this study are included in this article. Further enquiries can be directed to the corresponding author.

\section{References}

1 Biondi NL, Varacallo M. Enchondroma. In: StatPearls [Internet]. Treasure Island (FL): StatPearls Publishing; 2020 [cited 2021 Jan 11]. Available from: http://www.ncbi.nlm.nih.gov/books/NBK536938/.

2 Davies AM, Shah A, Shah R, Patel A, James SL, Botchu R. Are the tubular bones of the hand really the commonest site for an enchondroma? Clin Radiol. 2020 Jul;75(7):533-7.

3 Omlor GW, Lohnherr V, Lange J, Gantz S, Merle C, Fellenberg J, et al. Enchondromas and atypical cartilaginous tumors at the proximal humerus treated with intralesional resection and bone cement filling with or without osteosynthesis: retrospective analysis of 42 cases with 6 years mean follow-up. World J Surg Oncol. 2018 Dec;16(1):139.

4 Deckers C, Schreuder BHW, Hannink G, de Rooy JWJ, van der Geest ICM. Radiologic follow-up of untreated enchondroma and atypical cartilaginous tumors in the long bones: the natural course of chondroid tumors. J Surg Oncol. 2016 Dec;114(8):987-91.

5 Errani C, Tsukamoto S, Ciani G, Akahane M, Cevolani L, Tanzi P, et al. Risk factors for local recurrence from atypical cartilaginous tumour and enchondroma of the long bones. Eur J Orthop Surg Traumatol. 2017 Aug; 27(6):805-11.

6 Bart Schreuder HW, Pruszczynski M, Veth RPH, Lemmens JAM. Treatment of benign and low-grade malignant intramedullary chondroid tumours with curettage and cryosurgery. Eur J Surg Oncol. 1998 Apr;24(2):120-6.

\section{Karger's}


7 Hong ED, Carrino JA, Weber KL, Fayad LM. Prevalence of shoulder enchondromas on routine MR imaging. Clin Imaging. 2011 Sep;35(5):378-84.

8 Ferrer-Santacreu EM, Ortiz-Cruz EJ, González-López JM, Pérez Fernández E. Enchondroma versus low-grade chondrosarcoma in appendicular skeleton: clinical and radiological criteria. J Oncol. 2012;2012:437958.

9 Murphey MD, Flemming DJ, Boyea SR, Bojescul JA, Sweet DE, Temple HT. Enchondroma versus chondrosarcoma in the appendicular skeleton: differentiating features. Radiographics. 1998 Sep;18(5):1213-5.

10 Geirnaerdt MJ, Hermans J, Bloem JL, Kroon HM, Pope TL, Taminiau AH, et al. Usefulness of radiography in differentiating enchondroma from central grade 1 chondrosarcoma. Am J Roentgenol. 1997 Oct;169(4): 1097-104.

11 Bui KL, Ilaslan H, Bauer TW, Lietman SA, Joyce MJ, Sundaram M. Cortical scalloping and cortical penetration by small eccentric chondroid lesions in the long tubular bones: not a sign of malignancy? Skeletal Radiol. 2009 Aug;38(8):791-6.

12 Crim J, Schmidt R, Layfield L, Hanrahan C, Manaster BJ. Can imaging criteria distinguish enchondroma from grade 1 chondrosarcoma? Eur J Radiol. 2015 Nov;84(11):2222-30.

13 Eefting D, Schrage YM, Geirnaerdt MJA, Le Cessie S, Taminiau AHM, Bovée JVMG, et al. Assessment of interobserver variability and histologic parameters to improve reliability in classification and grading of central cartilaginous tumors. Am J Surg Pathol. 2009 Jan;33(1):50-7.

14 Brown MT, Gikas PD, Bhamra JS, Skinner JA, Aston WJS, Pollock RC, et al. How safe is curettage of low-grade cartilaginous neoplasms diagnosed by imaging with or without pre-operative needle biopsy? Bone Joint J. 2014 Aug;96-B(8):1098-105.

15 Mermerkaya M, Bekmez S, Karaaslan F, Danisman M, Kosemehmetoglu K, Gedikoglu G, et al. Intralesional curettage and cementation for low-grade chondrosarcoma of long bones: retrospective study and literature review. World J Surg Oncol. 2014;12(1):336.

16 Leal F, Nellensteijn JM, Frada R, Teixeira J, Queirós C, Padin M, et al. Arthroscopic treatment for femoral neck enchondroma: case report. J Hip Preserv Surg. 2015 Sep 1;2(4):428-30.

17 Laoruengthana A, Galassi M, Weerakul S, Assawaboonyadej P, Somran J. Result of arthroscopic removal of an enchondroma at the talar dome: a case report. J Med Assoc Thai. 2012 Oct;95 Suppl 10:S226-9.

18 Ando A, Koide M, Hagiwara Y, Matsuda M, Itoi E. Solitary bone cyst of the proximal humerus with a concomitant stiff shoulder treated with both arthroscopic capsular release and arthroscope-assisted resection of the bone cyst: a case report. Open J Orthop. 2018;8(3):77-84.

19 Couto A, Sá Rodrigues A, Nunes B, Torres J, Gutierres M. Arthroscopic approach to simple bone cyst of the humeral head-a step toward a minimally invasive technique. JSES Open Access. 2018 Dec;2(4):211-4.

20 Levy JC, Temple HT, Mollabashy A, Sanders J, Kransdorf M. The causes of pain in benign solitary enchondromas of the proximal humerus. Clin Orthop Relat Res. 2005 Feb;431:181-6. 